\title{
Correlatos Neuropsicológicos y Emocionales Implicados en el Consumo de Cocaína: Una Revisión Teórica a los Nuevos Hallazgos
}

\section{Neuropsychological and Emotional Correlates Involved in Cocaine Use: A Theoretical Review of New Findings}

\author{
Juan Ramos-Cejudo e Itziar Iruarrizaga Díez \\ Universidad Complutense de Madrid
}

\begin{abstract}
Resumen. El consumo de cocaína concierne un problema de salud alarmante en los países desarrollados. Se trata de un problema de importantes consecuencias a nivel económico, social y de salud pública. En la actualidad, los esfuerzos se centran en investigar los cambios metabólicos, neuropsicológicos y emocionales derivados del consumo crónico. Sin embargo, no contamos con trabajos de revisión teórica y crítica sobre éste ámbito. El objetivo de nuestro trabajo es revisar e integrar los últimos hallazgos y resultados encontrados en cuanto a las alteraciones metabólicas, neuropsicológicas y emocionales en el abuso de cocaína. Los resultados encontrados muestran un importante deterioro de las vías dopaminérgicas del lóbulo frontal, tanto en el número de receptores como en la depleción del neurotransmisor; así como un importante déficit en función ejecutiva, en inhibición de respuesta, en atención y en memoria operativa. Además, los estudios muestran alteraciones en la capacidad para identificar emociones, en impulsividad, toma de decisiones, ansiedad y depresión.
\end{abstract}

Palabras clave: cocaína, alteraciones neuropsicológicas, emoción, revisión teórica.

\begin{abstract}
Cocaine is being an alarming health problem in developed countries. This issue has important implications in economic, social and public health. Currently, efforts are focused on research in metabolic, neuropsychological and emotional changes, resulting from chronic use. However, theoretical work review on this critical area is not available. The aim of our study is to review and integrate the ultimate findings and results in terms of metabolic abnormalities, neuropsychological and emotional abuse of cocaine. The results show a significant impairment of dopaminergic pathways in the frontal lobe, both in the number of recipient and depletion of the neurotransmitter, as well as an important executive function deficit in response inhibition in attention and working memory. In addition, alterations in the ability in identifying emotions, impulsiveness, decision making, anxiety and depression are proved in different studies.

Keywords: cocaine, neuropsychological impairment, emotion, theoretical review.
\end{abstract}

\section{Introducción}

El abuso de cocaína es un problema heterogéneo y de graves consecuencias a nivel mundial. No solo por

La correspondencia sobre ese artículo deberá dirigirse al primer autor, Dpto. Psicología Básica II (Procesos Cognitivos). Facultad de Psicología, Buzón 17.28223 Madrid.E-mail: juan-ramos@cop.es la alta prevalencia y el incremento de la misma en los últimos años, sino también por las consecuencias emocionales, psicológicas y sociales derivadas del mismo. Los datos más recientes apuntan a un incremento del consumo de cocaína en el Reino Unido y en menor medida, en Dinamarca, Alemania, España y Países Bajos (EMCDDA, 2008); diferentes indicado- 
res como la demanda de tratamiento, los exámenes toxicológicos de las víctimas de sobredosis y las incautaciones de drogas en nuestro país, hacen de éste problema una realidad preocupante. Algunos Estados miembros de la Unión Europea (UE) han experimentado en los últimos años un incremento sustancial de la demanda de tratamientos por consumo de cocaína, la cual en la actualidad incluso supera la demanda de tratamiento por consumo de opiáceos en ciertos países, regiones y ciudades. Asimismo, una proporción importante de los consumidores de opiáceos en tratamiento menciona la cocaína como droga secundaria, hecho que puede contribuir a empeorar sus problemas y dificultar el tratamiento. En muchos países, la cocaína también aparece en los análisis toxicológicos de un alto porcentaje de las muertes relacionadas con las drogas, normalmente en combinación con opiáceos y otras sustancias (EMCDDA, 2008).

A fecha de hoy, España se encuentra entre los países con prevalencias más altas de la UE, en todos los grupos de edad estudiados, tanto en el último mes, como en el último año como a lo largo de la vida de los sujetos (ver tabla 1) y la tendencia más alarmante es en los varones de entre 15-34 años, horquilla en la que el consumo de cocaína se halla principalmente concentrado.

La cocaína es un estimulante del sistema nervioso central (SNC) que se absorbe por todas las vías de administración, se distribuye rápidamente por todo el organismo y su vida media es de 50 minutos aproximadamente. Esnifada o por vía intravenosa (IV) se encuentran niveles de cocaína en el cerebro en tan solo 30 segundos, y fumada, solo tarda 5 segundos en tener efectos centrales. Se trata de un inhibidor de los procesos de recaptación tipo I, que aumenta la biodisponibilidad de noradrenalina (NA) y dopamina (DA) en la hendidura sináptica. Además, la cocaína bloquea la recaptación de serotonina (SE) y presenta una acción anestésica local, disminuyendo la permeabilidad de la célula a los iones de sodio $(\mathrm{Na}+)$.

Esta droga presenta diferentes acciones farmacológicas, las cuales, se sitúan en la base de los principales daños para la salud de sus consumidores: en el aparato cardiovascular, actuando en los receptores $\alpha$ у $\beta$ adrenérgicos; en la temperatura corporal, por aumento de la actividad muscular; en el SNC, provocando elevación del estado de ánimo, sensación de mayor energía y lucidez, disminución del apetito, insomnio, mayor rendimiento, hiperactividad motora, verbal e ideativa, alteraciones de la percepción, pseudoalucinaciones táctiles, auditivas, visuales, conducta estereotipada, bruxismo y movimientos compulsivos; y en el aparato respiratorio, con taquipneas, respiración irregular y posible parada respiratoria.

El cerebro es uno de los órganos más directamente afectados por el consumo crónico de cocaína, provocando a su vez un importante deterioro cardiovascular, afectando especialmente al nivel de receptores, con una repercusión severa en diferentes funciones cognitivas (Madoz-Gurpide, Ochoa Mangado, \& Martinez Pelegrin, 2009), así como diferentes alteraciones neuropsicológicas (Woicik et al., 2009) que más tarde analizaremos.

En los últimos años se ha dado un fuerte crecimiento de trabajos publicados sobre el abuso de cocaína en las principales fuentes documentales (ver tabla 2). Sin embargo, no es frecuente encontrar en las principales bases de datos, revisiones teóricas y/o críticas sobre éstos tópicos. Cuando buscamos información sobre éstos temas, encontramos fundamentalmente estudios experimentales, comparaciones entre grupo experimental y grupo control, y correlaciones significativas, a falta de un potente grupo de trabajos que reúnan y revisen el conocimiento, organizándolo e integrándolo.

Nuestra revisión incluye los estudios publicados en PubMed (desde 1992 a junio de 2009) y en PsycINFO (desde 1992 a junio de 2009) usando los términos "emocional disorders", "cocaine" y "neuropsychological" en el campo abstract y con un total de 318 artículos, incluyendo estudios con humanos y con animales. El objetivo de nuestro trabajo es integrar los últimos hallazgos encontrados en el abuso crónico de cocaína, teniendo en cuenta los cambios metabólicos a nivel cerebral, las alteraciones neuropsicológicas y los procesos cognitivos y emocionales que quedan deteriorados en este tipo de adicción.

\section{Alteraciones metabólicas y sistemas de neuro- transmisión afectados}

El daño cerebral más obvio es consecuencia del efecto vascular directo de la sustancia (Madoz- 
Tabla 1: prevalencia del consumo de cocaína en los países de la Unión Europea. Con permiso del EMCDDA, 2008

\begin{tabular}{|c|c|c|c|}
\hline \multirow[t]{2}{*}{ Grupo de edad } & \multicolumn{3}{|c|}{ Período de tiempo del consumo } \\
\hline & Toda la vida & Último año & Último mes \\
\hline \multicolumn{4}{|l|}{ 15-64 años } \\
\hline $\begin{array}{l}\text { Número estimado } \\
\text { de consumidores en Europa }\end{array}$ & 12 millones & 4 millones & 2 millones \\
\hline Media europea & $3,6 \%$ & $1,2 \%$ & $0,5 \%$ \\
\hline Intervalo & $0,4-7,7 \%$ & $0,1-3,0 \%$ & $0-1,6 \%$ \\
\hline Países con con prevalencia más baja & $\begin{array}{l}\text { Rumanía, Malta, } \\
\text { Lituania, } \\
\text { Grecia }\end{array}$ & $\begin{array}{l}\text { Grecia, } \\
\text { Polonia, Letonia, } \\
\text { República Checa }\end{array}$ & $\begin{array}{l}\text { Grecia, Estonia, } \\
\text { República Checa, } \\
\text { Malta, Lituania, Polonia, } \\
\text { Finlandia, Letonia }\end{array}$ \\
\hline Países con prevalencia más alta & $\begin{array}{l}\text { Reino Unido }(7,7 \%) \\
\text { España }(7,0 \%) \\
\text { Italia }(6,6 \%) \\
\text { Irlanda }(5,3 \%)\end{array}$ & $\begin{array}{l}\text { España }(3,0 \%) \\
\text { Reino Unido }(2,6 \%) \\
\text { Italia }(2,2 \%) \\
\text { Irlanda }(1,7 \%)\end{array}$ & $\begin{array}{l}\text { España }(1,6 \%) \\
\text { Reino Unido }(1,3 \%) \\
\text { Italia }(0,8 \%) \\
\text { Irlanda }(0,5 \%)\end{array}$ \\
\hline \multicolumn{4}{|l|}{ 15-34 años } \\
\hline $\begin{array}{l}\text { Número estimado } \\
\text { de consumidores en Europa }\end{array}$ & 7,5 millones & 3,5 millones & 1,5 millones \\
\hline Media europea & $5,4 \%$ & $2,3 \%$ & $1 \%$ \\
\hline Intervalo & $0,7-12,7 \%$ & $0,2-5,4 \%$ & $0.0-2,8 \%$ \\
\hline Países con prevalencia más baja & $\begin{array}{l}\text { Rumania, Lituania } \\
\text { Malta } \\
\text { Grecia }\end{array}$ & $\begin{array}{l}\text { Grecia } \\
\text { Polonia } \\
\text { Letonia, República Checa }\end{array}$ & $\begin{array}{l}\text { Estonia } \\
\text { Grecia, Polonia, Letonia } \\
\text { República Checa }\end{array}$ \\
\hline Países con prevalencia más alta & $\begin{array}{l}\text { Reino Unido }(12,7 \%) \\
\text { España }(9,6 \%) \\
\text { Dinamarca }(9,1 \%) \\
\text { Irlanda }(8,2 \%)\end{array}$ & $\begin{array}{l}\text { Reino Unido }(5,4 \%) \\
\text { España }(5,2 \%) \\
\text { Italia }(3,2 \%) \\
\text { Irlanda }(3,1 \%)\end{array}$ & $\begin{array}{l}\text { España }(2,8 \%) \\
\text { Reino Unido }(2,7 \%) \\
\text { Italia }(1,2 \%) \\
\text { Dinamarca, Irlanda }(1,0 \%)\end{array}$ \\
\hline \multicolumn{4}{|l|}{ 15-24 años } \\
\hline $\begin{array}{l}\text { Número estimado } \\
\text { de consumidores en Europa }\end{array}$ & 3 millones & 2 millones & 800.000 \\
\hline Media europea & $4,5 \%$ & $2,6 \%$ & $1,2 \%$ \\
\hline Intervalo & $0,4-11,2 \%$ & $0,2-6,1 \%$ & $0,0-3,2 \%$ \\
\hline Países con prevalencia más baja & $\begin{array}{l}\text { Rumanía } \\
\text { Grecia } \\
\text { Lituania } \\
\text { Malta, Polonia }\end{array}$ & $\begin{array}{l}\text { Grecia } \\
\text { Polonia } \\
\text { República Checa } \\
\text { Letonia }\end{array}$ & $\begin{array}{l}\text { Estonia } \\
\text { Grecia, Letonia } \\
\text { República Checa, Polonia, } \\
\text { Portugal }\end{array}$ \\
\hline Países con prevalencia más alta & $\begin{array}{l}\text { Reino Unido }(11,2 \%) \\
\text { España }(8,7 \%) \\
\text { Dinamarca }(8,0 \%) \\
\text { Irlanda }(7,0 \%)\end{array}$ & $\begin{array}{l}\text { Reino Unido }(6,1 \%) \\
\text { España }(5,8 \%) \\
\text { Irlanda }(3,8 \%) \\
\text { Dinamarca, Italia }(3,3 \%)\end{array}$ & $\begin{array}{l}\text { Reino Unido }(3,2 \%) \\
\text { España }(3,1 \%) \\
\text { Italia }(1,3 \%) \\
\text { Bulgaria, Irlanda }(1,1 \%)\end{array}$ \\
\hline
\end{tabular}

Información basada en la última encuesta realizada en cada país. El estudio abarca del año 2001 al año 2007. Para obtener la prevalencia media en Europa se ha calculado una media en función de la población del grupo de edad relevante en cada país. A los países de losque nos e dispone de información se les ha aplicado la prevalencia media del a Unín Europea. Población usada como base: 15-64 años (328 millones), 15-34 años (134 millones) y 15-24 años (64 millones). Los datos aquí resumidos están disponibles en las «Encuestas de población general» del boletín estadístico de 2008 .. 
Figura 3: Número de trabajos publicados en PubMed utilizando las palabras clave "neuropsychological" y "cocaine" en el campo abstract

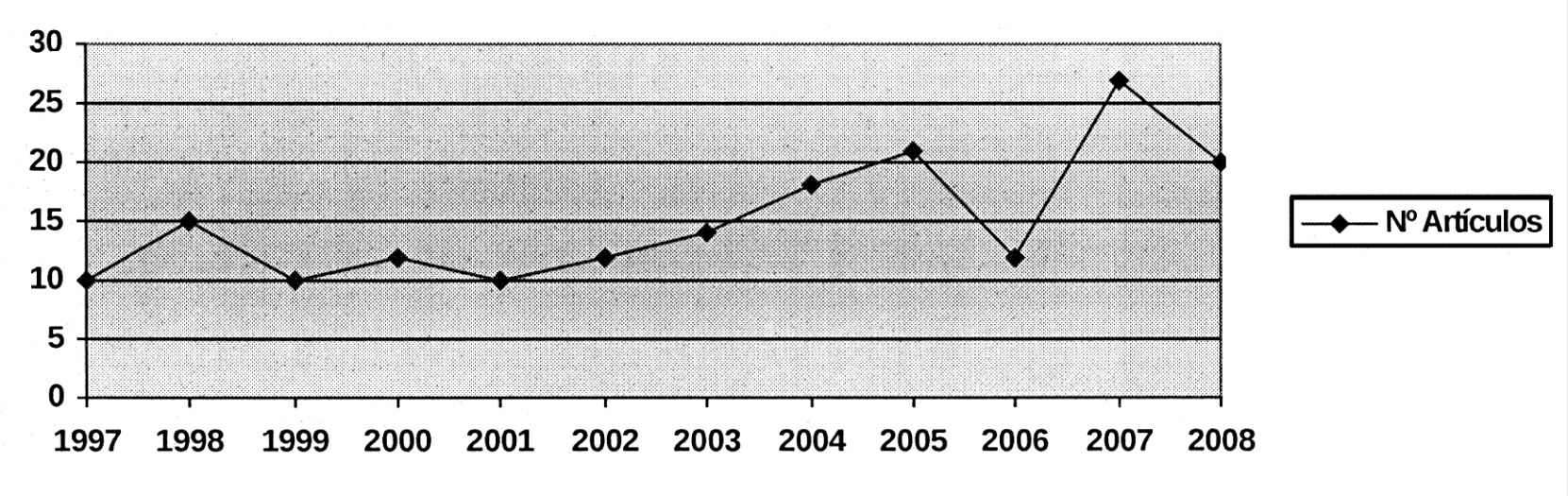

1 Inglaterra y País de Gales.

NB: En Dinamarca, el valor hacia 1994 corresponde a drogas duras. Se calculan el GPS-14 en el boletín 2008 estadístico para la información más lejana. Fuentes: Reitox informes nacionales (2007), tomado de revisiones demográficas, relata artículos ar científicos.

Gurpide et al., 2009) (i.e. elevación de la presión arterial, isquemia) además de provocar daño metabólico neuronal alterando el equilibrio de los neurotransmisores y de los receptores (Ernst, Chang, Oropilla, Gustavson, \& Speck, 2000). El sistema dopaminérgico es el más habitualmente afectado en estos pacientes y en particular, las vías situadas en el lóbulo frontal (Volkow \& Fowler, 2000). Numerosos estudios señalan una importante afectación de ésta corteza, de manera particular, la atención se ha centrado en analizar las relaciones existentes entre la corteza prefrontal y la afectación de las funciones ejecutivas en este tipo de pacientes (A. Verdejo-García, Pérez-García, Sánchez-Barrera, Rodríguez-Fernández, \& GómezRío, 2007).

Según estudios recientes (Madoz-Gurpide et al., 2009), existen tres regiones especialmente implicadas, a nivel clínico, en los consumidores de cocaína: el cortex orbitofrontal, que media en los efectos de recompensa de la sustancia, así como en los comportamientos impulsivos y en la toma de decisiones (Volkow \& Fowler, 2000); la corteza dorsolateral, que integra la información sensorial que procede del sistema límbico, esto es, nuestra vida emocional básica, organización de acciones, planificación de tareas, resolución de problemas y memoria operativa; y la corteza paralímbica, que nos viene a aportar el elemento motivacional. Estas tres regiones quedan ampliamente afectadas en el abuso de cocaína (Goldstein et al., 2007; MadozGurpide et al., 2009).

\section{Alteraciones neuropsicológicas}

Estos daños a nivel metabólico y de los sistemas de neurotransmisión afectados en los consumidores de cocaína, tienen una repercusión en las funciones neuropsicológicas de los pacientes, quedando deterioradas algunas de las funciones más básicas. Un claro reflejo de su deterioro se observa en el rendimiento bajo las pruebas neuropsicologícas administradas. De forma resumida, los deterioros más relevantes se sitúan en cuatro áreas fundamentales: procesos atencionales, memoria operativa, inhibición de respuesta y función ejecutiva.

En cuanto a los procesos atencionales, las revisiones y los estudios experimentales apuntan a fallos en atención selectiva y mantenida (Cunha, Nicastri, Gomes, Moino, \& Peluso, 2004; Goldstein et al., 2004; Gooding, Burroughs, \& Boutros, 2008; Horner, 1999; Jovanovski, Erb, \& Zakzanis, 2005; Woicik et al., 2009), así como mayor tasa de errores en tareas Stroop (Hester, Simoes-Franklin, \& Garavan, 2007). Especialmente en los periodos de abstinencia, se encuentran déficit en atención selectiva y sostenida (Abi-Saab et al., 2005; Bushnell et al., 2000; Copersino et al., 2004; Garavan \& Hester, 2007; Horner, 1999), e incluso cuando se da una exposición prenatal a la droga (Accornero et al., 2007), tanto en humanos (Heffelfinger, Craft, White, \& Shyken, 2002) como en modelos animales (He, Bai, Champoux, Suomi, \& Lidow, 2004).

En cuanto al déficit en memoria operativa y capacidad de aprendizaje, en un estudio reciente con una 
amplia muestra (Goldstein et al., 2004), se encontró un deterioro importante en memoria visual y verbal en consumidores de cocaína cuando se les administraba una batería neuropsicológica. Otros estudios han confirmado estos hallazgos encontrando importantes déficit en memoria operativa en dependientes (Ford, Farah, Shera, \& Hurt, 2007; Jovanovski et al., 2005; Kubler, Murphy, \& Garavan, 2005; Verdejo, Orozco-Gimenez, Meersmans Sanchez-Jofre, Aguilar de Arcos, \& Perez-Garcia, 2004), como en sujetos bajo periodos de abstinencia (Pace-Schott et al., 2008; Pace-Schott et al., 2005), como en sujetos que fueron expuestos de forma prenatal a la droga (Mayes, Snyder, Langlois, \& Hunter, 2007).

En lo referente al déficit en inhibición de respuesta, encontramos fallos importantes en sujetos consumidores de cocaína, en su mayoría, cuando realizan tareas de Go/No Go (Colzato \& Hommel, 2009; Fillmore \& Rush, 2006; Garavan \& Hester, 2007; Hester et al., 2007; A. Verdejo-Garcia \& PerezGarcia, 2007).

En un estudio reciente de meta-análisis realizado por Jovanovski et al. (2005) basándose en 15 estudios empíricos, se estudió el tamaño del efecto de los mismos, reuniendo a 481 sujetos consumidores de cocaína y 586 sujetos sanos (Jovanovski et al., 2005). Los resultados apuntan en la dirección de que los consumidores crónicos de cocaína presentan déficit importantes en atención $(0.40<\mathrm{d}<1.10)$, efectos moderados en memoria visual y operativa (d $>0.50$ ), y efectos leves en test de fluencia verbal y otras funciones senso-perceptivas $(\mathrm{d}<0.30)$.

Las funciones ejecutivas son un conjunto de habilidades de orden superior de organización e integración asociadas a la corteza prefrontal entre las que se incluyen: la capacidad de anticipación, el establecimiento de metas, el diseño de planes, la autorregulación, la ejecución, la inhibición de conductas, el juicio y razonamiento abstracto y la formación de conceptos (Spinella, 2003). Si revisamos los estudios más recientes, encontramos fallos en impulsividad y habilidades motoras (A. Verdejo-Garcia, Bechara, Recknor, \& Perez-Garcia, 2006; A. Verdejo-Garcia, A. Benbrook et al., 2007; A. Verdejo-Garcia \& Perez-Garcia, 2007) y en toma de decisiones cuando se les compara con sujetos control sanos (Stout, Busemeyer, Lin, Grant, \& Bonson, 2004). Además, la capacidad para la inhibición de conductas, la flexibilidad para el cambio, el procesamiento de errores y el manejo de contingencias quedan fuertemente deteriorados en estos pacientes (A. VerdejoGarcia \& Perez-Garcia, 2007).

\section{Alteraciones emocionales y psicopatología}

Ya en un estudio clásico, se encontraron diferencias estadísticamente significativas entre consumidores de cocaína y no consumidores, tanto en salud física, como en variables sociodemográficas implicadas y en salud mental (Newcomb \& Bentler, 1986). Incluso en algunos trabajos, ya antiguos, se han estudiado algunos factores de buen pronóstico en el tratamiento de deshabituación a la cocaína, como el estilo de afrontamiento y algunos factores de personalidad (McMahon, Kelley, \& Kouzekanani, 1993).

Una de las estructuras cerebrales implicadas en el consumo de cocaína es la corteza anterior del cíngulo (ACC). Los sujetos dependientes de cocaína presentan una importante hipoactivación de la ACC frente a los sujetos sanos (Goldstein et al., 2009) seguido de un deterioro conductual importante, cuando realizan las tareas cognitivas y emocionales. Una de las explicaciones más recientes que se han dado es que los sujetos que han vivido acontecimientos vitales estresantes importantes en la infancia (i.e. maltrato, negligencia paterna, etc.) aumentan la probabilidad de que se genere una irregularidad o degeneración en el eje hipotalámico-pituitario-adrenal (HPA), provocando así una predisposición al abuso de sustancias como la cocaína (Gerra et al., 2009). Para estos autores, los factores estresantes en la infancia, incluyendo la negligencia paterna, pueden afectar a los rasgos de personalidad de los individuos y a la salud mental, induciendo cambios estables en el HPA, implicando una vulnerabilidad a la conducta adictiva.

Una de las tareas utilizadas para ver los efectos emocionales tras el consumo crónico de cocaína es la identificación de expresiones emocionales faciales y la identificación de imágenes emocionales frente a imágenes neutras mediante el International Affective Picture System, IAPS (Lang, Bradley, \& 
Cuthbert, 1998). En un estudio reciente (VerdejoGarcia et al., 2006) se observó un procesamiento anormal de las imágenes afectivas del IAPS por parte de los sujetos adictos a cocaína, frente a los sujetos sanos. Además, otras investigaciones apuntan a una importante dificultad para identificar emociones en las expresiones faciales presentadas. Incluso, los consumidores ocasionales de cocaína, son lentos en identificar el asco y los consumidores regulares muestran un déficit importante en el reconocimiento del miedo (Kemmis, Hall, Kingston, \& Morgan, 2007).

Otra rama de estudios se centran en las consecuencias emocionales de la exposición prenatal a la droga, confirmando alteraciones en regulación emocional y activación emocional en los sujetos expuestos a la droga, así como una mayor sensibilidad al estrés y a la sintomatología fisiológica asociada al mismo (Stanger et al., 1999).

Por otro lado, numerosos estudios transversales han encontrado comorbilidad entre los sujetos consumidores de cocaína con diferentes trastornos mentales del Eje I (i.e. trastornos de ansiedad, del estado de ánimo) (Chaplin, Fahy, Sinha, \& Mayes, 2009; Herrero, Domingo-Salvany, Torrens, \& Brugal, 2008) y con trastornos por el control de los impulsos, como la tricotilomanía (Sanju \& Hamdy, 2005).

\section{Conclusiones}

En la línea de los resultados encontrados, dentro de las principales fuentes documentales en ésta área del conocimiento, nuestras conclusiones, en términos generales, no pueden ser halagüeñas. El consumo de cocaína en los países de la UE ha sufrido un potente incremento situándose en unas tasas a la cabeza a nivel mundial (EMCDDA, 2008). En este marco preocupante, España se sitúa como el principal país de Europa en inicio del consumo en todas sus horquillas de edad.

Los correlatos neuroanatómicos y neurofuncionales encontrados por los diferentes estudios de forma general, marcan un importante déficit en las vías dopaminérgicas situadas fundamentalmente en el lóbulo frontal (A. Verdejo-Garcia, M. Perez-Garcia et al., 2007; Volkow \& Fowler, 2000). El daño meta- bólico, tanto en el número de receptores como en los procesos de neurotransmisión, tiene que ver con los efectos directos de la sustancia (Ernst et al., 2000); esto es, quedan afectadas las vías que sufren cambios durante la intoxicación aguda (i.e. nivel de DA).

Los déficit neuropsicológicos que presentan los sujetos que han estado expuestos a la droga, tanto en consumo directo, como a nivel prenatal, van a estar asociados a los daños metabólicos sufridos. Es por eso que se encuentran déficit en función ejecutiva e inhibición de respuesta (Accornero et al., 2007; Colzato \& Hommel, 2009; Sheinkopf et al., 2009; A. J. Verdejo-Garcia, Perales, \& Perez-Garcia, 2007), en los procesos atencionales (Garavan \& Hester, 2007; Gooding et al., 2008; Kubler et al., 2005; Savage, Brodsky, Malmud, Giannetta, \& Hurt, 2005; Wittmann, Leland, Churan, \& Paulus, 2007; Woicik et al., 2009), y en memoria de trabajo (Goldstein et al., 2004; Woicik et al., 2009).

Además, se han encontrado problemas emocionales y alteraciones psicopatológicas (Ochoa 2000), como incapacidad para reconocer emociones faciales, niveles elevados de impulsividad con dificultades para la toma de decisiones (Stout et al., 2004) y síntomas depresivos asociados al consumo crónico. Posiblemente derivados del daño sufrido en áreas de la corteza frontal y en estructuras subcorticales implicadas en el procesamiento emocional (i.e. ACC, vías hipotálamo-amigdalares), confirman una vez más la relación existente entre tejido dañadoconducta alterada.

Esto supone un importante gasto en los sistemas de Salud Pública y la necesidad de adecuación de programas de tratamiento dentro de los recursos sanitarios públicos y privados. A pesar de los esfuerzos por dar con los fármacos ideales para el tratamiento de éste problema, los tratamientos de elección para los problemas de abuso de cocaína son intervenciones psicológicas cognitivo-conductuales (EMCDDA, 2008). Por otro lado, los profesionales que trabajan en éste tipo de adicciones, con frecuencia abordan el consumo de sustancias y tienen que intervenir sobre otros problemas psicopatológicos implicados o comórbiles en los pacientes (Becoña et al., 2008). Es por ello, que el conocimiento de las alteraciones neuropsicológicas y neurofuncionales debe tenerse en cuenta para la adecuación de nues- 
tras intervenciones. La implantación de las técnicas cognitivo-conductuales deberá tener en cuenta los déficit neuropsicológicos, neurofuncionales y emocionales que presentarán los pacientes.

\section{Referencias}

Abi-Saab, D., Beauvais, J., Mehm, J., Brody, M., Gottschalk, C., \& Kosten, T. R. (2005). The effect of alcohol on the neuropsychological functioning of recently abstinent cocaine-dependent subjects. Am J Addict, 14, 166-178.

Accornero, V. H., Amado, A. J., Morrow, C. E., Xue, L., Anthony, J. C., \& Bandstra, E. S. (2007). Impact of prenatal cocaine exposure on attention and response inhibition as assessed by continuous performance tests. J Dev Behav Pediatr, 28, 195-205.

Becoña, E., Cortés, M., Pedrero, E. J., Fernández, J. R., Casete, L., Bermejo, M. P. et al. (2008). Guía clínica de intervención psicológica en adicciones. Guías clínicas Socidrogalcohol basadas en la evidencia científica. Recogida el 10 de junio de 2009 en http://socidrogalcohol.psiquiatria.com/documentos/guia_sda_intervenciones_psicologicas.pdf

Bushnell, P. J., Levin, E. D., Marrocco, R. T., Sarter, M. F., Strupp, B. J., \& Warburton, D. M. (2000). Attention as a target of intoxication: insights and methods from studies of drug abuse. Neurotoxicol Teratol, 22, 487-502.

Chaplin, T. M., Fahy, T., Sinha, R., \& Mayes, L. C. (2009). Emotional arousal in cocaine exposed toddlers: Prediction of behavior problems. Neurotoxicol Teratol, 31, 275-282.

Colzato, L. S., \& Hommel, B. (2009). Recreational use of cocaine eliminates inhibition of return. Neuropsychology, 23, 125-129.

Copersino, M. L., Serper, M. R., Vadhan, N., Goldberg, B. R., Richarme, D., Chou, J. C., et al. (2004). Cocaine craving and attentional bias in cocaine-dependent schizophrenic patients. Psychiatry Res, 128, 209-218.

Cunha, P. J., Nicastri, S., Gomes, L. P., Moino, R. M., \& Peluso, M. A. (2004). [Neuropsychological impairments in crack cocaine-dependent inpatients: preliminary findings]. Rev Bras Psiquiatr, 26, 103-106.
Ernst, T., Chang, L., Oropilla, G., Gustavson, A., \& Speck, O. (2000). Cerebral perfusion abnormalities in abstinent cocaine abusers: a perfusion MRI and SPECT study. Psychiatry Res, 99, 63-74.

European and Monitoring Center for Drugs and Drugs Addiction (2008). Informe 2008 del Observatorio Europeo Sobre Drogas. Recogido el 15 de junio de 2009 en http://www.pnsd.msc.es/Categoria2/publica/pdf/oed-2008.pdf.

Fillmore, M. T., \& Rush, C. R. (2006). Polydrug abusers display impaired discrimination-reversal learning in a model of behavioural control. $J$ Psychopharmacol, 20, 24-32.

Ford, S., Farah, M. S., Shera, D. M., \& Hurt, H. (2007). Neurocognitive correlates of problem behavior in environmentally at-risk adolescents. $J$ Dev Behav Pediatr, 28, 376-385.

Garavan, H., \& Hester, R. (2007). The role of cognitive control in cocaine dependence. Neuropsychol Rev, 17, 337-345.

Gerra, G., Leonardi, C., Cortese, E., Zaimovic, A., Dell'agnello, G., Manfredini, M., et al. (2009). Childhood neglect and parental care perception in cocaine addicts: relation with psychiatric symptoms and biological correlates. Neurosci Biobehav Rev, 33, 601-610.

Goldstein, R. Z., Alia-Klein, N., Tomasi, D., Carrillo, J. H., Maloney, T., Woicik, P. A., et al. (2009). Anterior cingulate cortex hypoactivations to an emotionally salient task in cocaine addiction. Proc Natl Acad Sci U S A, 106, 9453-9458.

Goldstein, R. Z., Leskovjan, A. C., Hoff, A. L., Hitzemann, R., Bashan, F., Khalsa, S. S., et al. (2004). Severity of neuropsychological impairment in cocaine and alcohol addiction: association with metabolism in the prefrontal cortex. Neuropsychologia, 42, 1447-1458.

Goldstein, R. Z., Tomasi, D., Rajaram, S., Cottone, L. A., Zhang, L., Maloney, T., et al. (2007). Role of the anterior cingulate and medial orbitofrontal cortex in processing drug cues in cocaine addiction. Neuroscience, 144, 1153-1159.

Gooding, D. C., Burroughs, S., \& Boutros, N. N. (2008). Attentional deficits in cocaine-dependent patients: converging behavioral and electrophysiological evidence. Psychiatry Res, 160, 145-154.

He, N., Bai, J., Champoux, M., Suomi, S. J., \& 
Lidow, M. S. (2004). Neurobehavioral deficits in neonatal rhesus monkeys exposed to cocaine in utero. Neurotoxicol Teratol, 26, 13-21.

Heffelfinger, A. K., Craft, S., White, D. A., \& Shyken, J. (2002). Visual attention in preschool children prenatally exposed to cocaine: implications for behavioral regulation. $J$ Int Neuropsychol Soc, 8, 12-21.

Herrero, M. J. Â. s., Domingo-Salvany, A., Torrens, M., \& Brugal, M. T. (2008). Addiction, 103, 284293.

Hester, R., Simoes-Franklin, C., \& Garavan, H. (2007). Post-error behavior in active cocaine users: poor awareness of errors in the presence of intact performance adjustments. Neuropsychopharmacology, 32, 1974-1984.

Horner, M. D. (1999). Attentional functioning in abstinent cocaine abusers. Drug Alcohol Depend, 54, 19-33.

Jovanovski, D., Erb, S., \& Zakzanis, K. K. (2005). Neurocognitive deficits in cocaine users: a quantitative review of the evidence. J Clin Exp Neuropsychol, 27, 189-204.

Kemmis, L., Hall, J. K., Kingston, R., \& Morgan, M. J. (2007). Impaired fear recognition in regular recreational cocaine users. Psychopharmacology (Berl), 194, 151-159.

Kubler, A., Murphy, K., \& Garavan, H. (2005). Cocaine dependence and attention switching within and between verbal and visuospatial working memory. Eur J Neurosci, 21, 1984-1992.

Lang, P. J., Bradley, M. M., \& Cuthbert, B. N. (1998). Emotion and motivation: measuring affective perception. J Clin Neurophysiol, 15, 397-408.

Madoz-Gurpide, A., Ochoa Mangado, E., \& Martinez Pelegrin, B. (2009). [Use of cocaine and neuropsychological damage. Clinical implications.]. Med Clin (Barc), 132, 555-559.

Mayes, L., Snyder, P. J., Langlois, E., \& Hunter, N. (2007). Visuospatial working memory in schoolaged children exposed in utero to cocaine. Child Neuropsychol, 13, 205-218.

McMahon, R. C., Kelley, A., \& Kouzekanani, K. (1993). Personality and coping styles in the prediction of dropout from treatment for cocaine abuse. J Pers Assess, 61, 147-155.

Newcomb, M. D., \& Bentler, P. M. (1986). Cocaine use among young adults. Adv Alcohol Subst Abuse, 6, 73-96.

Ochoa, E. (2000). Cocaína y comorbilidad psiquiátrica. Actas Españolas de Psiquiatría, 28, 40-52.

Pace-Schott, E. F., Morgan, P. T., Malison, R. T., Hart, C. L., Edgar, C., Walker, M., et al. (2008). Cocaine users differ from normals on cognitive tasks which show poorer performance during drug abstinence. Am J Drug Alcohol Abuse, 34, 109-121.

Pace-Schott, E. F., Stickgold, R., Muzur, A., Wigren, P. E., Ward, A. S., Hart, C. L., et al. (2005). Cognitive performance by humans during a smoked cocaine binge-abstinence cycle. Am J Drug Alcohol Abuse, 31, 571-591.

Sanju, G., \& Hamdy, M. (2005). Cocaine-induced trichotillomania. Addiction, 100, 255-256.

Savage, J., Brodsky, N. L., Malmud, E., Giannetta, J. M., \& Hurt, H. (2005). Attentional functioning and impulse control in cocaine-exposed and control children at age ten years. J Dev Behav Pediatr, 26, 42-47.

Sheinkopf, S. J., Lester, B. M., Sanes, J. N., Eliassen, J. C., Hutchison, E. R., Seifer, R., et al. (2009). Functional MRI and response inhibition in children exposed to cocaine in utero. Preliminary findings. Dev Neurosci, 31, 159-166.

Spinella, M. (2003). Relationship between drug use and prefrontal-associated traits. Addict Biol, 8(1), 67-74.

Stanger, C., Higgins, S. T., Bickel, W. K., Elk, R., Grabowski, J., Schmitz, J., et al. (1999). Behavioral and emotional problems among children of cocaine- and opiate-dependent parents. J Am Acad Child Adolesc Psychiatry, 38, 421-428.

Stout, J. C., Busemeyer, J. R., Lin, A., Grant, S. J., \& Bonson, K. R. (2004). Cognitive modeling analysis of decision-making processes in cocaine abusers. Psychon Bull Rev, 11, 742-747.

Verdejo, A., Orozco-Gimenez, C., Meersmans Sanchez-Jofre, M., Aguilar de Arcos, F., \& PerezGarcia, M. (2004). [The impact exerted by the severity of recreational drug abuse on the different components of the executive function]. Rev Neurol, 38, 1109-1116.

Verdejo-Garcia, A., Bechara, A., Recknor, E. C., \& Perez-Garcia, M. (2006). Executive dysfunction 
in substance dependent individuals during drug use and abstinence: an examination of the behavioral, cognitive and emotional correlates of addiction. J Int Neuropsychol Soc, 12, 405-415.

Verdejo-Garcia, A., Benbrook, A., Funderburk, F., David, P., Cadet, J. L., \& Bolla, K. I. (2007). The differential relationship between cocaine use and marijuana use on decision-making performance over repeat testing with the Iowa Gambling Task. Drug Alcohol Depend, 90, 2-11.

Verdejo-Garcia, A., \& Perez-Garcia, M. (2007). Profile of executive deficits in cocaine and heroin polysubstance users: common and differential effects on separate executive components. Psychopharmacology (Berl), 190, 517-530.

Verdejo-Garcia, A., Perez-Garcia, M., SanchezBarrera, M., Rodriguez-Fernandez, A., \& GomezRio, M. (2007). [Neuroimaging and drug addiction: neuroanatomical correlates of cocaine, opi- ates, cannabis and ecstasy abuse]. Rev Neurol, 44, 432-439.

Verdejo-Garcia, A. J., Perales, J. C., \& Perez-Garcia, M. (2007). Cognitive impulsivity in cocaine and heroin polysubstance abusers. Addict Behav, 32(5), 950-966.

Volkow, N. D., \& Fowler, J. S. (2000). Addiction, a disease of compulsion and drive: involvement of the orbitofrontal cortex. Cereb Cortex, 10, 318325.

Wittmann, M., Leland, D. S., Churan, J., \& Paulus, M. P. (2007). Impaired time perception and motor timing in stimulant-dependent subjects. Drug Alcohol Depend, 90, 183-192.

Woicik, P. A., Moeller, S. J., Alia-Klein, N., Maloney, T., Lukasik, T. M., Yeliosof, O., et al. (2009). The neuropsychology of cocaine addiction: recent cocaine use masks impairment. Neuropsychopharmacology, 34, 1112-1122.

Manuscrito recibido: 15/09/2009

Revisión recibida: 06/10/2009

Manuscrito aceptado: 08/10/2009 